\title{
LA HABILIDAD DE CUIDADO DEL CUIDADOR FAMILIAR EN DIFERENTES REGIONES DE COLOMBIA
}

\section{CARING ABILITY OF FAMILY CAREGIVER IN DIFFERENT COLOMBIAN REGIONS}

Lorena Chaparro-Díaz ${ }^{*}$, Sonia Patricia Carreño², María Stella Campos-de-Aldana ${ }^{3}$, Fanny Benavides ${ }^{4}$, Carmen Lucía NiñoCardozo $^{5}$, Ruby Mercedes Cardona ${ }^{6}$, María Luisa Pinzón ${ }^{7}$, Olga Marina Vega ${ }^{8}$, María Lucenith Criado $^{9}$

${ }^{1}$ Enfermera, Doctora en Enfermería, Profesora Asociada Universidad Nacional de Colombia - Sede Bogotá - Facultad de Enfermería- Grupo de Investigación Cuidado de Enfermería al paciente crónico - Carrera 30 No.45 - 03, Edif. 228, Bogotá, Código Postal: 111321 - Colombia. e-mail: olchaparrod@unal.edu.co; ${ }^{2}$ Enfermera, Magister y Doctora en Enfermería, Profesora Auxiliar Universidad Nacional de Colombia - Sede Bogotá - Facultad de Enfermería- Grupo de Investigación Cuidado de Enfermería al paciente crónico - Colombia. e-mail spcarrenom@unal.edu.co; ${ }^{3}$ Enfermera, especialista en Gerencia Social, especialista en Salud Ocupacional. Magister en Enfermería en Salud Familiar. Docente Titular Programa de Enfermería Universidad de Santander (UDES), e-mail: scamposaldana@hotmail.com; cuidadoenfermeria@udes.edu.co; ${ }^{4}$ Enfermera, Especialista en educación. Profesora Titular, Universidad Mariana. e-mail: fannypatriciaba@gmail.com; ${ }^{5}$ Enfermera. Magister en Bioética. Especialista en Atención de Enfermería al Paciente Crítico. Docente Programa de Enfermería de la Universidad de Ciencias Aplicadas y Ambientales U.D.C.A. e-mail: cnino@udca.edu.co; ${ }^{6}$ Enfermera. Maestrante de ciencias de la educación. Especialista en medico quirúrgica con énfasis en UCI. Directora de programa de enfermería Universidad Tecnológica del Chocó “Diego Luis Córdoba”. e-mail: rubyc_12@hotmail.com; ${ }^{7}$ Enfermera. Magister en Enfermería con énfasis en Cuidado Crónico, Magister en Desarrollo educativo y social. Decana Facultad Ciencias de la Salud, Universidad de los Llanos. e-mail: Marialuisa.pinzon@unillanos.edu.co; ${ }^{8}$ Enfermera. Magister en Enfermería Cuidado al Paciente Crónico. Docente titular, Programa de Enfermería, Universidad Francisco de Paula Santander. e-mail: olgavega@ufps.edu.co; ${ }^{9}$ Enfermera. Magister en Enfermería con énfasis en Salud Familiar. Coordinadora de Investigación Faculta de Ciencias de la Educación y de la Salud de la Fundación Universitaria de San Gil UNISANGIL. mcriado@unisangil.edu.co; *Autora para correspondencia

Rev. U.D.C.A Act. \& Div. Cient. 19(2): 275-284, Julio-Diciembre 2016

\section{RESUMEN}

La habilidad de cuidado es un fenómeno de interés en salud, que es susceptible de abordar, como indicador de la atención. Existen algunas similitudes y diferencias reportadas en Colombia y en América Latina, que indican una heterogeneidad; sin embargo, no existe un diagnóstico nacional contundente. El objetivo fue describir el nivel de habilidad de cuidado del cuidador familiar de personas con enfermedad crónica no transmisible (ECNT), en todas las regiones geográficas de Colombia y la relación con las características de los cuidadores. Es un estudio cuantitativo, descriptivo y correlacional. La muestra fue de 2.551 cuidadores familiares. Se utilizó la encuesta de caracterización de la diada y el inventario de habilidad de cuidado de Nkongho. Se encontró mediana y baja habilidad de cuidado en los cuidadores familiares; las dimensiones mejor valoradas fueron conocimiento y valor, mientras que la paciencia fue la más baja. Las regiones geográficas de Colombia presentaron niveles de habilidad similares, excepto la Amazonía, que reveló altos niveles de habilidad en todas las dimensiones.
Se encontró una relación lineal negativa entre la habilidad de cuidado con la edad y el estrato y relación lineal positiva entre el tiempo y la habilidad. Se requieren intervenciones educativas para fortalecer la habilidad de cuidado de los cuidadores familiares de personas con enfermedad crónica en todas sus dimensiones, especialmente, en la paciencia.

Palabras clave: Enfermedad crónica, familia, aptitud.

\section{SUMMARY}

The caring ability is a relevant phenomenon in health field, which can be addressed as healthcare indicator. In Colombia and Latin America, some similarities and differences that indicate heterogeneity do exist, nevertheless, a forceful diagnosis at the national scale is absent. The objective was to describe the caring ability level of the family caregiver of patients with Non communicable Diseases (NCDs) in all Colombian regions in relation to characteristics of caregivers; a quantitative, descriptive, correlational study was carried out. The sample comprised 2551 family 
caregivers. The characterization of the dyad survey, and the caring ability inventory, developed by Nkongho, were used. Medium and low caring ability level was found. The slightly affected dimensions were knowledge and value, whereas the patience dimension was the lowest. Geographic regions of Colombia showed similar patterns, except for the Colombian Amazonia, which showed high levels in all the dimensions. A negative linear relationship between caring ability with age and socioeconomic level, and a positive linear relationship between time and ability was found. Educational interventions are needed to strengthen caring ability of the family caregiver of people with chronic disease in all the dimensions, particularly in patience.

Key words: Chronic illness, family, aptitude.

\section{INTRODUCCIÓN}

Las Enfermedades Crónicas no Transmisibles (ECNT) han venido en crecimiento y son uno de los temas de interés en salud mundial. De acuerdo con la Organización Mundial de la Salud (OMS) $(2013$; 2014) en el mundo, el 63\% de la mortalidad general es atribuible a este tipo de enfermedades, 36 millones de personas mueren cada año y cerca del $80 \%$ ocurren en países de ingresos medios y bajos, entre los que se encuentra Colombia.

De acuerdo con Barrera et al. (2010), la ECNT afectan a quien la padece, generándole diferentes grados de limitación en su funcionalidad, lo que hace que requiera apoyo de un cuidador familiar. Rogero (2010) afirma que el cuidador debe asumir diversas tareas, para las cuales, podría no estar preparado. La experiencia de cuidar a la persona con ECNT y la complejidad que engloba el cuidado brindado crea sobrecarga, según Goldstein et al. (2003) y Zambrano \& Ceballos (2007); ocasiona, según Björk et al. (2005), cambios en el ámbito laboral y financiero; disminuye la calidad de vida, según Braun et al. (2007), Bellin et al. (2013) y Guiven et al. (2012) y genera sentimientos de angustia y de temor, de acuerdo con Flury et al. (2011).

El cuidador demanda apoyo en esta experiencia y requiere potencializar las fortalezas individuales. Una de éstas, es la habilidad de cuidado, que ha sido estudiada en varias ciudades de Colombia y de América Latina, a partir de lo planteado por Nkongho (2003), quien reconoce la habilidad de cuidado, como el potencial de cuidado que tienen las personas en situaciones adversas, como la cronicidad de un familiar. Se aborda a partir de la dimensión cognoscitiva y actitudinal y se miden según los componentes de conocimiento, de valor y de paciencia.

Para Nkongho (2003), el conocimiento implica entender quién es la persona que se cuida e incluye tomar conciencia de las propias fortalezas y limitaciones; el valor, se relaciona con la identificación de una red de apoyo y con la forma de tomar decisiones y la paciencia, hace referencia a dar tiempo y espacio para la autoexpresión y exploración y se relaciona con encontrar significado.

Los estudios anteriores fueron realizados por Ramírez (2005) y González (2006), en Norte de Santander; Díaz (2007), en Girardot; Barrera et al. (2006b) y (2006c), en Chía y Villavicencio; Montalvo et al. (2010), en Cartagena y, más recientemente, por Gélvez et al. (2014), Carrillo et al. (2014b; 2015), en Bogotá. A nivel de América Latina, específicamente en México, han abordado la habilidad de cuidado Marín et al. (2011), Tizoc et al. (2012) y Ostiguín-Meléndez et al. (2012). Se requiere hacer una descripción global que se constituya en línea base para orientar intervenciones de impacto en Colombia y en sus regiones.

El objetivo del presente estudio fue detallar el nivel de habilidad de cuidado del cuidador familiar de personas con ECNT, en todas las regiones de Colombia y su relación con aspectos característicos de los cuidadores.

\section{MATERIALES Y MÉTODOS}

El presente es un estudio de tipo cuantitativo, descriptivo, correlacional de corte transversal. Se desarrolló en el 2014, en el marco del Programa para la Disminución de la Carga de la Enfermedad Crónica en Colombia, en el que participaron diferentes nodos de la Red Latinoamericana de Cuidado al Paciente Crónico y la Familia, entre los que se encuentran Universidad Nacional de Colombia, Universidad de Ciencias Aplicadas y Ambientales U.D.C.A, Universidad de Santander, Universidad Mariana de Pasto -Departamento Nariño-, Fundación Universitaria de San Gil -Departamento Santander-, Universidad de los Llanos -Departamento Meta-, Universidad Tecnológica del Chocó -Departamento Chocó- y la Universidad Francisco de Paula Santander -Departamento Norte de Santander-.

La muestra estuvo constituida por 2.551 cuidadores familiares de personas con ECNT, en las cinco regiones de Colombia, que propone el Instituto Geográfico Agustín Codazzi: Amazonía, Andina, Caribe, Pacífico y Orinoquía y que coinciden con las características socioculturales definidas por Gutiérrez de Pineda (1975), como tipologías de las familias colombianas (Tabla 1). Se tuvo en cuenta la elaboración de una muestra maestra, es decir, un esquema matricial análogo a un cuadrado latino, basado en submuestras independientes de base común de comparación (intencional), la cual, se realizó a partir de los nodos vinculados al proyecto, en donde cada nodo definía los puntos de observación (instituciones hospitalarias o comunitarias) y se recolectaron los datos, en un periodo de tiempo de 12 meses. 
Tabla 1. Distribución de la muestra por región.

\begin{tabular}{|c|c|c|}
\hline REGIÓN & NÚMERO DE CUIDADORES & PORCENTAJE \\
\hline Amazonía & $\mathbf{2 0 0}$ & $\mathbf{7 , 8}$ \\
\hline Andina & 2.098 & $\mathbf{8 2 , 2}$ \\
\hline Caribe & 86 & 3,3 \\
\hline Pacífico & 77 & 3,01 \\
\hline Orinoquía & 90 & 3,52 \\
\hline TOTAL & $\mathbf{2 . 5 5 1}$ & $\mathbf{1 0 0 \%}$ \\
\hline
\end{tabular}

Los criterios de inclusión de los cuidadores familiares eran: cuidar una persona con ECNT por tiempo mayor a un mes, tener un vínculo de cercanía o parentesco con la persona con ECNT, mayor de 18 años de edad, sin dificultades para responder los instrumentos.

La vinculación en el estudio fue voluntaria y se aplicó el consentimiento informado. Este estudio fue avalado por el Comité de Ética de la Facultad de Enfermería de la Universidad Nacional de Colombia y de las universidades que hicieron parte de la Red, que contaban con Comité de Ética; se consideró lo establecido en la Resolución 008430 de 1993, que reglamenta la investigación en Colombia (República de Colombia, MinSalud, 1993) y las pautas éticas internacionales, para la investigación biomédica en seres humanos (CIOMS et al. 2012).

Para la recolección de la información, se utilizó la encuesta de caracterización de la diada, persona con enfermedad de crónica-cuidador familiar, planteada por Chaparro et al. (2014). La Encuesta consta de 42 ítems y es válida para ser aplicada en el contexto colombiano. Para el presente estudio, se emplearon los ítems correspondientes a la caracterización sociodemográfica; también, se usó el inventario de habilidad de cuidado de Nkongho (2003), válido y confiable en el contexto colombiano. Este instrumento está constituido por 37 ítems, con respuesta tipo Likert, que van de 1 a 7 , 1 equivale a totalmente en desacuerdo y 7 totalmente de acuerdo. Contiene tres subescalas: Conocimiento, con 14 ítems; valor, con 13 y paciencia, con 10 . El puntaje total está entre mínimo 37 y máximo 259, con puntos de corte para habilidad de cuidado alta (220,3 o más), media $(203,1-220,2)$ y baja (203 o menos). Para los componentes que la integran: Conocimiento, mínimo 14 y máximo 98 - habilidad de cuidado alta (84 o más), media (76,4-83) y baja (76,3 o menos); valor, con mínimo 13 y máximo 91 - habilidad de cuidado alta (74 o más), media (62,5-73) y baja (62,4 o menos) y paciencia, mínimo 10 y máximo 70 - habilidad de cuidado alta $(65,2$ o más), media $(61-65,1)$ y baja (60 o menos).

La versión original tiene validez de contenido y reporta una confiabilidad por consistencia interna alfa de Cronbach para cada factor, de 0,71 a 0,84. En la versión en español, el instrumento fue sometido a afinación semántica y a pruebas de validez y de confiabilidad, por parte de la Unión Temporal - UT carga de la enfermedad crónica, en el marco del programa para la disminución de la carga del cuidado de la enfermedad crónica, encontrando que es válido y confiable para ser usado en el contexto colombiano, en cuidadores de personas con enfermedad crónica. El test - re test reportó un coeficiente de correlación de Pearson de 0,80 y alpha de Cronbach, para cada una de las aplicaciones, con valores mayores o iguales 0,89 , lo cual, indica que es confiable (aplicación 1 test: 0,89; aplicación 2 re-test: 0,93).

Se utilizó estadística descriptiva para el análisis de los datos por región y total y correlación de Pearson, entre la habilidad de cuidado y sus componentes, con algunas características sociodemográficas. Para este análisis, se usó el programa SPSS versión 21, bajo la licencia de la Universidad Nacional de Colombia.

\section{RESULTADOS Y DISCUSIÓN}

Los cuidadores colombianos son en su mayoría mujeres, en edades altamente productivas para la sociedad, con medianos y bajos niveles de escolaridad, viven en zonas urbanas, de ocupación hogar y en estratos socioeconómicos medio y bajo. Este perfil reafirma lo reportado en el contexto colombiano por Carrillo et al. (2014b), López et al. (2015) y Carrillo et al. (2015), en América Latina, por Ostiguín-Meléndez et al. (2012), Eterovic et al. (2015) y, a nivel internacional, por Crespo-López \& LópezMartínez (2008) y Juratovac \& Zauszniewski (2014), lo que indica, una condición de alta vulnerabilidad social por el poco reconocimiento social y pobres políticas dirigidas al mejoramiento de su desarrollo individual y calidad de vida.

De acuerdo con Gaugle et al. (2008), los cuidadores presentan una sobrecarga en cuanto al tiempo dedicado en el cuidado y la limitada disponibilidad de apoyo familiar, lo que impide vinculación laboral estable y es más visible en las mujeres cuidadoras (Tabla 2). 
Tabla 2. Características del cuidador.

\begin{tabular}{|c|c|c|c|}
\hline Ítem & Categoría & Número & Porcentaje \\
\hline \multirow[t]{2}{*}{ Género } & Femenino & 2.110 & 82,7 \\
\hline & Masculino & 441 & 17,3 \\
\hline \multirow[t]{4}{*}{ Edad en años } & De 18 a 35 & 1.122 & 44,0 \\
\hline & De 36 a 59 & 1.006 & 39,4 \\
\hline & De 60 a 75 & 325 & 12,7 \\
\hline & Más de 75 & 98 & 3,8 \\
\hline \multirow[t]{9}{*}{ Grado máximo de escolaridad } & Analfabeta & 23 & 0,9 \\
\hline & Primaria Incompleta & 421 & 16,5 \\
\hline & Primaria Completa & 365 & 14,3 \\
\hline & Bachillerato Incompleto & 563 & 22,1 \\
\hline & Bachillerato Completo & 296 & 11,6 \\
\hline & Técnico & 358 & 14,0 \\
\hline & Universidad Incompleta & 234 & 9,2 \\
\hline & Universidad Completa & 268 & 10,5 \\
\hline & Posgrados & 23 & 0,9 \\
\hline \multirow[t]{2}{*}{ Lugar de residencia } & Rural & 416 & 16,3 \\
\hline & Urbana & 2.135 & 83,7 \\
\hline \multirow[t]{5}{*}{ Estado Civil } & Casado(a) & 754 & 29,6 \\
\hline & Separado(a) & 182 & 7,1 \\
\hline & Soltero(a) & 793 & 31,1 \\
\hline & Unión Libre & 728 & 28,5 \\
\hline & Viudo(a) & 94 & 3,7 \\
\hline \multirow[t]{5}{*}{ Ocupación } & Empleado(a) & 596 & 23,4 \\
\hline & Estudiante & 189 & 7,4 \\
\hline & Hogar & 1.054 & 41,3 \\
\hline & Trabajo Independiente & 614 & 24,1 \\
\hline & Otra. ¿Cuál? & 98 & 3,8 \\
\hline \multirow[t]{6}{*}{ Estrato socioeconómico } & 1 & 1.245 & 48,8 \\
\hline & 2 & 885 & 34,7 \\
\hline & 3 & 364 & 14,3 \\
\hline & 4 & 49 & 1,9 \\
\hline & 5 & 6 & 0,2 \\
\hline & 6 & 2 & 0,1 \\
\hline \multirow[t]{2}{*}{ ¿Es usted el único cuidador? } & No & 1.206 & 47,3 \\
\hline & Si & 1.345 & 52,7 \\
\hline \multirow[t]{4}{*}{ Tiempo que lleva como cuidador } & 0 a 6 meses & 304 & 11,9 \\
\hline & 19 a 36 meses & 306 & 12,0 \\
\hline & 7 a 18 meses & 428 & 16,8 \\
\hline & Más de 37 meses & 1.513 & 59,3 \\
\hline \multirow[t]{3}{*}{ Dedicación diaria al cuidado en horas } & 6 o menos & 1.270 & 49,8 \\
\hline & 7 a 12 & 523 & 20,5 \\
\hline & 13 a 24 & 758 & 29,7 \\
\hline
\end{tabular}


Aunque el cuidado se considera una labor innata en los seres humanos, en donde la retribución familiar juega un papel importante, existe un gran riesgo de abandono, si no se cuenta con estrategias que reconozcan al cuidador y fortalezcan el vínculo con su familiar, tal como lo sugieren Fulmer et al. (2005). Es importante ofrecer apoyo instrumental y social en periodos de descanso o de forma permanente, según lo planteado por Jeon et al. (2005).

El nivel de habilidad de cuidado del cuidador, de acuerdo con Lindqvist et al (2004) existe la posibilidad que, para afrontar la situación, tenga influencia de género y, por lo tanto, recomienda que se valoren y se midan estas habilidades.

La habilidad de cuidado de los cuidadores colombianos muestra alta variabilidad, especialmente, para la región Pacífica, Caribe y Andina; sin embargo, las regiones Orinoquia y Amazonía presentan los mejores puntajes en la habilidad de cuidado general, siendo esta última la de mejor habilidad en el país (Tabla 3).

Tabla 3. Descripción de la habilidad de cuidado total por región.

\begin{tabular}{|l|c|c|c|c|c|}
\hline Región & Media & Mediana & Desviación Estándar & Mínimo & Máximo \\
\hline Orinoquía & 215,28 & 215 & 12 & 185 & 247 \\
\hline Amazonia & 246,85 & 259 & 23 & 148 & 259 \\
\hline Caribe & 193,67 & 208 & 32 & 82 & 245 \\
\hline Andina & 202,2 & 205 & 34 & 37 & 259 \\
\hline Pacífico & 196,23 & 212 & 49 & 37 & 259 \\
\hline Colombia & 205,69 & 210 & 40 & & \\
\hline
\end{tabular}

Los resultados generales son similares a lo reportado en los últimos cinco años (Carrillo et al. 2014b; OstiguínMeléndez et al. 2012; López et al. 2015), lo que indica que los cuidadores tienen muy poco reconocimiento de su rol, por la disminución de su potencial -como ser humano-, para ser cuidador y resignificarse, lo que genera mayor carga manifestada en poca satisfacción y sentimientos negativos de la experiencia.

En cuanto a la clasificación de la habilidad de cuidado en Colombia, en general, hay una predominancia hacia medio y bajo (63\%); sin embargo, las regiones que presentan mejor habilidad son la Amazonia y medianamente la Orinoquía; las regiones Caribe, Andina y Pacífico son las de menor habilidad (Tabla 4).

En cuanto a los niveles en la dimensión de la habilidad de cuidado, la Amazonía presenta una puntación con criterio alto (220,3 o más de acuerdo a la escala), en todas las dimensiones. El conocimiento, se encuentra afectado en las regiones costeras del país, lo que indica que los cuidadores se reconocen como cuidadores, pero existen dificultades asociadas, probablemente, a la falta de apoyo familiar y reconocimiento del rol en la carga del cuidado.

Tabla 4. Clasificación de la habilidad de cuidado por niveles bajo, medio y alto en las regiones.

\begin{tabular}{|l|l|l|l|l|l|l|l|l|l|l|l|l|l|}
\hline & \multicolumn{3}{|c|}{ BAJO } & \multicolumn{2}{c|}{ MEDIO } & \multicolumn{2}{c|}{ ALTO } & \multicolumn{2}{c|}{ Conocimiento } & \multicolumn{3}{c|}{ Valor } & \multicolumn{2}{c|}{ Paciencia } \\
\hline REGIÓN & \multicolumn{1}{|c}{ N. } & $\mathbf{\%}$ & $\mathbf{N .}$ & $\mathbf{\%}$ & $\mathbf{N .}$ & $\mathbf{\%}$ & Puntaje & Categoría & Puntaje & Categoría & Puntaje & Categoría \\
\hline Orinoquía & 15 & 16 & 42 & 47 & 33 & 37 & 87,8 & Alto & 63,3 & Medio & 64,2 & Medio \\
\hline Amazonia & 13 & 6 & 3 & 2 & 184 & 92 & 93,6 & Alto & 86,8 & Alto & 66,4 & Alto \\
\hline Caribe & 42 & 49 & 31 & 36 & 13 & 15 & 74 & Bajo & 68 & Medio & 51,6 & Bajo \\
\hline Andina & 1.007 & 48 & 419 & 20 & 672 & 32 & 80 & Medio & 64,8 & Medio & 57,4 & Bajo \\
\hline Pacífico & 33 & 43 & 14 & 18 & 30 & 39 & 75,2 & Bajo & 68,3 & Medio & 52,7 & Bajo \\
\hline Colombia & 1.110 & 43 & 509 & 20 & 932 & 37 & 82,1 & Medio & 70,2 & Medio & 58,5 & Bajo \\
\hline
\end{tabular}


Por otro lado, el valor y la paciencia se encuentran afectados en todas las regiones, excepto en la Amazonía, lo que coincide con lo reportado por Ostiguín-Meléndez et al. (2012), Eterovic et al. (2015) y Tizoc et al. (2012), frente a mejores niveles de paciencia. Givens et al. (2012) reconocen que la carga del cuidado limita la toma de decisiones y genera sufrimiento emocional, lo que restringe, de forma importante, la trascendencia de la experiencia en la vida personal de los cuidadores y podría afectar su bienestar. Por otro lado, Sandy et al. (2013) afirman que las necesidades de apoyo de los cuidadores relacionadas con la red de apoyo, lo financiero, lo emocional y el acompañamiento en la labor, son elementos clave de los cuidadores familiares de niños con discapacidades físicas.

En la relación entre características sociodemográficas y la habilidad de cuidado, se tuvieron en cuenta las variables sociodemográficas de edad, grado máximo de escolaridad, estrato socioeconómico, tiempo que lleva como cuidador y número de horas que dedica diariamente al cuidado. Se buscó descartar la hipótesis teórica que el perfil del cuidador familiar colombiano, no influye en la habilidad de cuidado. Se utilizó la correlación de Pearson, para valores del inventario de habilidad de cuidado, con la conversión de los datos a variables numéricas (Tabla 5).

Se encontró que, a mayor edad de los cuidadores, existe una mejor habilidad. Los cuidadores jóvenes cuentan con habilidades de afrontamiento y toma de decisiones, que deben ser analizadas a la luz de las demandas de la adversidad actual de la vida, aspecto que es parte de una política pública, con el reconocimiento de cuidadores niños y jóvenes, tal como lo plantea Becker (2007).

Según Eterovic et al. (2015), los cuidadores de adultos presentan mayor variabilidad en su habilidad de cuidado general y los cuidadores de personas con discapacidad, mejores puntajes. Llama la atención para Marín et al. (2011), la baja habilidad de cuidado reportada en cuidadores de niños con cáncer, lo que indica la necesidad de atender a esta población, que va en aumento, por el patrón epidemiológico asociado.

Por otro lado, el estudio reportó que la habilidad para cuidar no se relaciona con el nivel de escolaridad. Estos resultados difieren de lo reportado por Tizoc et al. (2012), quien encontró que a mayor escolaridad menor habilidad. Los resultados del presente estudio son una contribución para la intervención, teniendo en cuenta los hallazgos en el nivel de escolaridad, se podrían aplicar estrategias grupales para mejorar, especialmente, el valor, porque la experiencia vivida es la misma. Como componente de la habilidad, la paciencia si se relacionó en el estudio de Ostiguín- Meléndez et al. (2012) con la escolaridad, lo cual, indica un posible riesgo en cuidadores analfabetas de enfrentarse al sufrimiento, así como la percepción negativa de la experiencia.

Pese a que el estrato socioeconómico se suele relacionar con la escolaridad, este estudio muestra una particularidad, porque indica que pertenecer a un estrato socioeconómico alto disminuye la capacidad de toma de decisiones, que se asocia con el valor, como componente de la habilidad de cuidado, lo que puede estar relacionado con mayor exposición al cuidado directo, en cuidadores de estratos bajos.

El tiempo de cuidado -en términos de cuánto lleva como cuidador-, establece una relación lineal con la habilidad de cuidado, es decir, a mayor tiempo de cuidado diario, mayor es la habilidad adquirida por los cuidadores, que coincide con lo reportado por Carrillo et al. (2014a).

Tabla 5. Correlación de variables sociodemográficas con habilidad de cuidado.

\begin{tabular}{|l|l|l|l|l|l|l|l|l|}
\hline \multirow{2}{*}{ VARIABLE SOCIODEMOGRÁFICA } & \multicolumn{2}{|c|}{ Conocimiento } & \multicolumn{2}{c|}{ Valor } & \multicolumn{2}{c|}{ Paciencia } & \multicolumn{2}{c|}{$\begin{array}{c}\text { Total habilidad de } \\
\text { cuidado }\end{array}$} \\
\cline { 2 - 9 } & \multicolumn{1}{|c|}{$\mathbf{R}$} & $\mathbf{P}$ & $\mathbf{R}$ & $\mathbf{P}$ & $\mathbf{R}$ & $\mathbf{1}$ & \multicolumn{1}{|c|}{$\mathbf{R}$} & \multicolumn{1}{|c|}{ P } \\
\hline Edad en años & $-0,116^{*}$ & 0,000 & $-0,13^{*}$ & 0,000 & $-0,124^{*}$ & 0,000 & $-0,127^{*}$ & 0,000 \\
\hline Grado máximo de escolaridad & $-0,032$ & 0,287 & $-0,041$ & 0,163 & $-0,033$ & 0,274 &,- 038 & 0,203 \\
\hline Estrato socioeconómico & $-0,047$ & 0,113 & $-0,071^{* *}$ & 0,016 & $-0,046$ & 0,119 &,- 058 & 0,050 \\
\hline Tiempo que lleva como cuidador & $0,268^{*}$ & 0,000 & $0,301^{*}$ & 0,000 & $0,206^{*}$ & 0,000 & $0,181^{*}$ & 0,000 \\
\hline $\begin{array}{l}\text { Número de horas que dedica } \\
\text { diariamente al cuidado }\end{array}$ & $0,168^{*}$ & 0,000 & $0,183^{*}$ & 0,000 & $0,164^{*}$ & 0,000 & $0,178^{*}$ & 0,000 \\
\hline
\end{tabular}

*Significancia estadística $\mathrm{p}<0,01$ (bilateral); **Significancia estadística $\mathrm{p}<0.05$ (bilateral). 
En el caso del componente de conocimiento, OstiguínMeléndez et al. (2012) y Carrillo et al. (2014b), hallaron esta dimensión medianamente afectada; sin embargo, este comportamiento difiere, según Carrillo et al. (2014b), en cuidadores de adultos y cuidadores de mujeres mastectomizadas. Adicionalmente, Ostiguín-Meléndez et al. (2012) encontraron buena capacidad para reconocer a quien se cuida y las potencialidades de ser cuidador. Las regiones de Colombia no reportaron diferencias importantes.

En Colombia, el componente valor es similar a lo reportado por Tizoc et al. (2012), en México y por Barrera et al. (2006a), en Argentina y en Guatemala. Se encontró, que los niveles medios de valor en la región Andina son similares a lo informado en Cúcuta, por Ramírez (2005) y González (2006), así como en Girardot, por Díaz (2007). En la región Orinoquía, se aprecian niveles medios al igual que en el estudio realizado en Villavicencio, por Barrera et al. (2006b). En la región Caribe, también se reportan niveles medios, similares al estudio de Montalvo et al. (2010), en Cartagena. En general, se observa que este componente tuvo puntajes medios en todas las regiones, excepto en la Amazonía, en donde se presentaron puntajes altos.

Por otro lado, la dimensión de valor, según Ostiguín-Meléndez et al. (2012), se ve medianamente afectada en situaciones de cuidado de una mujer mastectomizada; según Carrillo et al. (2015) y Marín et al. (2011) es similar en situaciones como cuidador de un niño con cáncer. Tizoc et al. (2012) presentan resultados similares en cuidadores de personas con enfermedad renal crónica y Eterovic et al. (2015), en cuidadores de personas con discapacidad.

El componente de paciencia presenta gran variabilidad en los estudios de Tizoc et al. (2012) y Marín et al. (2011), en México y Barrera et al. (2006a), en Argentina y en Guatemala, los cuales, reportan un nivel medio; sin embargo, se registraron niveles altos de paciencia, en el estudio de Ostiguín-Meléndez et al. (2012), en cuidadores de mujeres mastectomizadas, en México. En la región Andina, se reporta un nivel bajo de paciencia, similar a lo reportado en Cúcuta, por González (2006) y Ramírez (2005) y difiere con lo encontrado en Girardot, por Díaz (2007) y González (2006), en donde se halla un nivel medio. En la Orinoquía, se apreció un nivel medio, similar a lo reportado por Barrera et al. (2006b), en un estudio realizado en Villavicencio. En la región Caribe, se evidenció un nivel bajo, que difiere del estudio de Montalvo et al. (2010), en Cartagena, quien encontró niveles altos. Estos hallazgos pueden estar asociados a la cultura específica de la región, que genera valoración específica del significado y trascendencia de la labor.

Este estudio pone de manifiesto que la habilidad de cuidado presenta niveles deficientes en Colombia y sus regiones, excepto en la región de la Amazonía. Se requiere mayor investigación en las regiones de la Amazonía y el Caribe; en la primera, porque los resultados son discordantes con las demás regiones frente a aspectos que influyen en tener mejor habilidad para cuidar, quizás de tipo cultural o de afrontamiento a la labor de cuidador y, en la segunda, por encontrar niveles opuestos categorizados en alto y bajo, en la misma población.

Este diagnóstico nacional arroja y valida los datos reportados por la Red Latinoamericana de Cuidado al Paciente Crónico y la familia y otros investigadores sobre el perfil de los cuidadores, por lo que debe ser un insumo social para el reconocimiento político e institucional del cuidador.

Se concluye que la habilidad de cuidado del cuidador familiar colombiano es mediana y baja, lo cual, indica la necesidad de abordar el fenómeno con intervenciones que fortalezcan, no solo el conocimiento de la enfermedad de su familiar, sino que deben impactar sobre el reconocimiento de la labor, herramientas tangibles y precisas para tomar decisiones, sin sentir culpa y la posibilidad de reconocer los aspectos positivos de la experiencia de cuidar. El concepto de conocimiento debe ser revisado y abordado de forma integral, no centrarse solo en la competencia, tal como lo menciona Mohammed et al. (2015), sino en la habilidad de conocer en cada etapa del proceso de la ECNT; este puede ser el mejor direccionamiento para las intervenciones en cuidadores familiares.

Es importante mencionar que este diagnóstico coincide con otros estudios realizados en Colombia y algunos países de América Latina, pero también plantea hipótesis alrededor del cuidador joven, estrato socioeconómico, grado de escolaridad, tiempo de cuidado y las características de la cultura amazónica, que pueden ser aspectos para profundizar con otras investigaciones y que influyen en aspectos, como la toma de decisiones y la resignificación de la experiencia. En cuanto al tiempo de cuidado, se requiere de estudios longitudinales, que validen la hipótesis.

Es necesario realizar estudios adicionales que exploren aspectos del contexto que afectan la habilidad de cuidado en las regiones, con mejores porcentajes de población de cuidadores, teniendo en cuenta que este estudio se llevó a cabo con una muestra maestra, que contempla un esquema matricial análogo a un cuadrado latino, basado en submuestras, independientes de base común de comparación y no necesariamente una equiparación porcentual. Esta puede ser una limitación del estudio.

El sistema de salud colombiano no reconoce la labor del cuidador, aunque existen iniciativas aisladas, a través de las instituciones prestadoras de servicios de salud y se requiere 
retomar iniciativas de orden jurídico para el reconocimiento de la labor del cuidador, así como de sus necesidades.

El liderazgo de Colombia en el abordaje de la habilidad de cuidado de los cuidadores familiares ha generado un reconocimiento social importante a la labor de estos actores del sistema de salud; no hay puntos de comparación a nivel internacional anglosajón o europeo, en cuanto a la habilidad de cuidado, que sirvan de referencia internacional, dando una oportunidad de establecimiento de redes internacionales.

Agradecimientos: Los investigadores expresan su agradecimiento a los participantes, instituciones y resto de profesionales y estudiantes, quienes apoyaron el desarrollo del estudio en diferentes modalidades. Conflicto de Intereses: El presente manuscrito fue preparado y revisado con la participación de todas las autoras, quienes declaramos que no existe conflicto de intereses que ponga en riesgo la validez de los resultados presentados. Financiación: Este estudio fue desarrollado dentro del marco del Programa para la Disminución de la Carga de la Enfermedad Crónica no Transmisible en Colombia. Colciencias Convocatoria 537 de 2011 código 110153730890, Código Hermes: 7872

\section{BIBLIOGRAFÍA}

1. BARRERA, L.; BLANCO, L.; FIGUEROA, P.; PINTO, N.; SÁNCHEZ, B. 2006a. Habilidad de cuidadores familiares de personas con enfermedad crónica, Mirada internacional. Aquichan (Colombia). 6(1):2233.

2. BARRERA, L.; GALVIS, C.; MORENO, M.; PINTO, N.; PINZÓN, M.; ROMERO, E.; SANCHEZ, B. 2006b. La habilidad de cuidado de los cuidadores familiares de personas con enfermedad crónica. Un estudio comparativo de tres unidades académicas de enfermería. Rev. Invest. Educ. Enfermería. (Colombia). 24(1):36-41.

3. BARRERA, L.; PINTO, N.; SÁNCHEZ, B. 2006c. Habilidad de cuidado de cuidadores familiares de personas con enfermedad crónica: comparación de géneros. Actualizaciones en Enfermería. (Colombia). 9(2):9-13.

4. BARRERA, L.; PINTO, N.; SÁNCHEZ, B.; CARRILLO, G.; CHAPARRO, L. 2010. Cuidando a los cuidadores Familiares de personas con enfermedad crónica. Colombia: U. Nacional de Colombia. 360p.

5. BECKER, S. 2007. Global Perspectives on Children's Unpaid Caregiving in the Family. Global Social Policy. 7(1):23-50.
6. BELLIN, M.; KUB, J.; FRICK, K.; BOLLINGER, M.; TSOUKLERIS, M.; WALKER, J.; LAND, C.; BUTZ, A. 2013. Stress and quality of life in caregivers of innercity minority children with poorly controlled asthma. J. Pediatric Health Care. (USA). 27(2):127-134.

7. BJÖRK, M.; WIBE, T.; HALLSTRÖM, I. 2005. Striving to survive: families' lived experiences when a child is diagnosed with cancer. J. Pediatic Oncology Nursing. (USA). 22(5):265-275.

8. BRAUIN, M.; MIKULINCER, M.; RYDALL, A.; WALSH, A.; RODIN, G. 2007. Hidden morbidity in cancer: spouse caregivers, J. Clinical Oncology. (USA). 25(30):48294832.

9. CARRILlO, G.; CHAPARRO, L; SÁNCHEZ, B. 2014a. Carga del cuidado en cuidadores familiares de personas con enfermedad crónica en la región pacífica colombiana. Ciencia y Enfermería. (Chile). 20(2):83-91.

10. CARRILLO, G.; SáNCHEZ, B.; BARRERA, L. 2014b. Habilidad de cuidado de cuidadores familiares de niños y adultos con enfermedad crónica. Index Enfermería. (España). 23(3):129-33.

11. CARRILLO, G.; SÁNCHEZ, B.; BARRERA, L. 2015. Habilidad de cuidado de cuidadores familiares de niños con cáncer. Rev. Salud Pública. (Colombia). 17(3):394-403.

12. CHAPARRO, L.; SÁNCHEZ, B.; CARRILLO, G. 2014. Encuesta de caracterización del cuidado de la diada cuidador familiar-persona con enfermedad crónica. Rev. Ciencia y Cuidado. (Colombia). 11(2):31-45.

13. CIOMS; OMS. 2012. Pautas éticas internacionales para la investigación biomédica en seres humanos preparadas por el consejo de organizaciones internacionales de las ciencias médicas (CIOMS) en colaboración con la Organización Mundial de la Salud (OMS), Ginebra, p.26-40, 72-75.

14. CRESPO-LÓPEZ, M.; LÓPEZ-MARTÍNEZ, J. 2008. Cuidadoras y cuidadores: el efecto del género en el cuidado no profesional de los mayores. Bol. sobre Envejecimiento. Perfiles y Tendencias. No. 35. $1^{\text {a }}$ ed. [Internet]. Madrid: IMSERSO. Disponible desde internet en: http://www.imserso.es/interpresent3/ groups/imserso/documents/binario/boletinopm35. pdf (con acceso 15/04/2016). 
15. DÍAZ, J. 2007. Habilidades de cuidado de los cuidadores familiares de personas en situación de enfermedad crónica vinculados al hospital San Rafael de Girardot Family care provider's care capability. Avan. Enfermería. (Colombia). 25(1):69-82.

16. ETEROVIC, C.; MENDOZA, S.; SÁEZ, K. 2015. Habilidad de cuidado y nivel de sobrecarga en cuidadoras/es informales de personas dependientes. Enfermería Global versión On-line. (España). 14(38):235-248. Disponible desde internet en: http://revistas.um.es/ eglobal/article/viewFile/198121/174111 (con acceso 10/01/2016).

17. FLURY, M.; CAFLISCH, U.; ULLMANN-BREMI, A.; SPICHIGERM, E. 2011. Experiences of parents with caring for their child after a cancer diagnosis. J. Pediatric Oncology Nursing. 28(3):143-153.

18. FULMER, T.; PAVEZA, G.; VANDEWEERD, C.; FAIRCHILD, S.; GUADAGNO, L.; BOLTON-BLATT, M.; NORMAN, R. 2005. Dyadic vulnerability and risk profiling for elder neglect. The Gerontologist. 45(4):525-534.

19. GAUGLER, J.; GIVEN, W.; LINDER, J.; KATARIA, R.; TUCKER, G.; REGINE, W. 2008. Work, gender, and stress in family cancer caregiving. Supportive Care in Cancer. 16(4):347-357.

20. GÉLVEZ, J.; ARIAS, S.; CÁRDENAS V. 2014. Habilidad del cuidador de pacientes con patología crónica discapacitante en casa, Rev. Médica Sanitas. (Colombia). 17(1):15-23.

21. GIVENS, J.; LOPEZ, R.; MAZOR, K.; MITCHELL, S. 2012. Sources of stress for family members of nursing home residents with advanced dementia. Alzheimer Disease and Associated Disorders. (USA). 26(3):254-259.

22. GOLDSTEIN, N.E.; CONCATO, J.; FRIED, T.R.; KASL, S.V.; JOHNSON-HURZELER, R.; BRADLEY, E.H. 2003. Factors associated with caregiver burden among caregivers of terminally ill patients with cancer. J. Paliative Care. (Canada). 20(1):38-43.

23. GONZÁLEZ, D. 2006. Habilidad de cuidado de los cuidadores familiares de personas en situación de enfermedad crónica por diabetes Mellitus. Avances Enfermería. 24 (2):28- 37.

24. GUIVEN, B.; GUIVEN, C.; SHERWOOD, P. 2012. The challenge of quality cancer care for family caregivers.
Seminars in Oncology Nursing. (USA). 28(4):205212.

25. GUTIÉRREZ DE PINEDA, V. 1975. Familia y cultura en Colombia; tipologías, funciones y dinámica de la familia: Manifestaciones múltiples a través del mosaico cultural y sus estructuras sociales. Ed. U. de Antioquia. 528p.

26. JEON, Y.H.; BRODATY, H.; CHESTERSON, J. 2005. Respite care for caregivers and people with severe mental illness: literature review. J. Advanced Nursing. 49(3):297-306.

27. JURATOVAC, E.; ZAUSZNIEWSKI, J. 2014. A. Full-time employed and a family caregiver: A profile of women's workload. Effort and Health. 24(2):187-196.

28. LINDQVIST, G.; HÅKANSSON, A.; PETERSSON, K. 2004. Informal home caregiving in a gender perspective: A selected literature review. Vard Nord Utveckl Forsk. 24(4):26-30.

29. LÓPEZ, D.; RODRÍGUEZ, L.; CARREÑO, S.; CUENCA, I.; CHAPARRO, L. 2015. Cuidadores de pacientes en diálisis peritoneal: experiencia de participar en un programa de habilidad de cuidado. Enfermería Nefrológica. (España). 18(3):189-195.

30. MARÍN, G.; ALARCÓN, M.; RAMÍREZ, M. 2011. Habilidad de cuidado del cuidador informal del niño con leucemia. Enlaces Académicos. (México). 4(1):3843.

31. MOHAMMED, H.J.; KAMEL, A.A. 2015. The Effect of Home Caregiving Program for Family Members Providing Care for Chronically Ill Relative Client. J. Education Practice. 6(22):18-21.

32. MONTALVO, A.; BADRÁN, Y.; CAVADÍA, C.; MEDINA, E.; MÉNDEZ, K.; PADILLA, C.; RUIDÍAZ, K. 2010. Habilidad de cuidado de cuidadores familiares principales de pacientes con ACV. Rev. Cient. Salud Uninorte. 26(2):212-22.

33. NKONGHO, N. 2003. The caring ability inventory. En: Strickland, O.; Diloro, C. (eds). Measurement of nursing outcomes. USA. 3:184-98.

34. ORGANIZACIÓN MUNDIAL DE LA SALUD -OMS-. 2013. 10 datos sobre las enfermedades no transmisibles. Disponible desde Internet en: http://www.who.int/ features/factfiles/noncommunicable_diseases/es/ (con acceso 27/01/2016). 
35. OSTIGUIIN-MELÉNDEZ, R.M.; RIVAS- HERRERA, J.C.; VALLEJO- ALLENDE, M.; CRESPO- KNOPFLER, S.; ALVARADO- AGUILAR, S. 2012. Habilidades del cuidador primario de mujeres mastectomizadas. Invest. Educ. Enfermería. 30(1):9-17.

36. RAMÍREZ, M. 2005. Habilidad de cuidado de los cuidadores principales de pacientes con cáncer gastrointestinal. Avances en Enfermería. 23(2):7-17.

37. REPÚBLICA DE COLOMBIA -MINISTERIO DE SALUD-. 1993. Resolución $N^{\circ} 008430$ Por la cual se establecen las normas científicas, técnicas y administrativas para la investigación en salud. Disponible desde Internet en: https://www.minsalud.gov.co/Normatividad Nuevo/RESOLUCION\%208430\%20DE\%201993.pdf (con acceso 20/01/2014).

38. ROGERO, J. 2010. Las consecuencias del cuidado familiar sobre el cuidador: Una valoración compleja y necesaria, Index de Enfermería. (España). 19(1):4750.
39. SANDY, P.T.; KGOLE, J.C.; MAVUNDLA, T.R. 2013. Support needs of caregivers: case studies in South Africa. Int Nurs Rev. 60(39):344-350.

40. TIZOC, A.; UZETA, J.; PERAZA, M.; MEDRANO, F.; VALDEZ, S. 2012. Nivel de habilidad del cuidador familiar en el cuidado a pacientes con enfermedad renal crónica del servicio UNEME del hospital general de Culiacán, Rev. Médica UAS. (México). 3(2):14-22.

41. WORLD HEALTH ORGANIZATION. 2014. Noncommunicable diseases country profiles 2014. Suiza: WHO Library Cataloguing in Publication Data. 207p.

42. ZAMBRANO, R.; CEBALLOS, P. 2007. Síndrome de carga del cuidador. Rev. Col. Psiquiatría. 36:26-39.

Recibido: Febrero 3 de 2016

Aceptado: Octubre 14 de 2016

Cómo citar:

Chaparro-Díaz, L.; Carreño, S.P.; Campos-de-Aldana, M.S.; Benavides, F.; Niño-Cardozo, C.L.; Cardona, R.M.; Pinzón, M.L.; Vega, O.M.; Criado, M.L. 2016. La habilidad de cuidado del cuidador familiar en diferentes regiones de Colombia. Rev. U.D.C.A Act. \& Div. Cient. 19(2): 275-284. 